\title{
Wealth and health in relation to birth defects mortality
}

Infant mortality is associated with poverty. Countries with higher per capita income have lower infant mortality rates. ${ }^{1}$ The most obvious reason for this is the burden of infant deaths attributable to potentially avoidable diseases, such as infectious diseases for which vaccines and drugs are available, but are not affordable for all. In rich countries, besides gross national product per head, income inequality is an important determinant of infant mortality rates. ${ }^{2}$ Countries with less income inequality have a lower infant mortality. This suggests that poverty remains an important determinant of infant mortality, even in relatively rich countries.

In Western countries infant mortality and morbidity are no longer dominated by infectious diseases and nutritional problems, unlike in many developing countries. The relative contribution of birth defects has therefore increased. While mortality attributable to communicable diseases, maternal and perinatal conditions and nutritional deficiencies is more than five times as frequent in low and middle income countries, as compared with high income countries, for congenital abnormalities these mortality figures differ only by a factor $2 .^{1}$

So far, birth defects remained a group of disorders for which little evidence was available to show that increased wealth was associated with decreased mortality and morbidity. Neural tube defects were reported to show an association with socioeconomic class, especially in Great Britain. ${ }^{3}$ Furthermore, the periconceptional use of folic acid to prevent neural tube defects is associated with socioeconomic class. ${ }^{4}$ Therefore, there were some indications that, also in the field of birth defects, preventable mortality, associated with poverty, exists.

In this issue of the fournal of Epidemiology and Community Health, in an impressive paper Rosano et al present data on infant mortality attributable to congenital anomalies in 36 countries. The paper is based on data from vital statistics reported to the World Health Organisation. Several questions to policy makers in the field of birth defects prevention can be raised after reading the paper and comparing the figures of one nation to the others. The most obvious example is infant mortality attributable to spina bifida, as preventive strategies are available. Should the contribution of spina bifida to infant mortality in a certain area be considerable, then clearly the policy concerning periconceptional use of folic acid or fortification of foods needs attention. ${ }^{5}$ Poverty should not necessarily be a threshold for such a preventive strategy, as folic acid is very cheap. Existing supplementation programmes for women of reproductive age, including iron for instance, could be expanded to folic acid.

Another issue concerning birth defects prevention is mentioned by Rosano et al in the discussion section of their paper. Some wealthier countries have extensive antenatal screening programmes for birth defects. Substantial numbers of selective abortions could reduce the number of affected infants born, and, if common enough, would result in a decline in infant mortality attributable to congenital anomalies. The parents concerned, however, have experienced the loss of an (often wanted) pregnancy. Low infant mortality attributable to congenital anomalies may reflect effective antenatal screening programmes, and thus may not always represent actual prevention.

A final question is whether low infant mortality rates are always good rates. With increasing wealth, increasing possibilities for treatment of infants with birth defects have become available. If the birth prevalence is stable, higher survival rates might lead to an increasing morbidity. The ultimate targets of birth defects prevention are both lower morbidity and lower mortality figures.

MARTINA C CORNEL Department of Medical Genetics, A Deusinglaan 4, NL-9713 AW Groningen, the Netherlands

1 World Health Organization. The world health report 1999. Making a difference. Geneva: WHO, 1999.

2 Hales S, Howden-Chapman P, Salmond C, et al. National infant mortality rates in relation to gross national product and distribution of income. Lancet 1999;354:2047.

3 Elwood JM, Little J, Elwood JH. Epidemiology and control of neural tube defects. Oxford: Oxford University Press, 1992.

4 De Walle HEK, Van der Pal KM, De Jong-Van den Berg LTW, et al. Effect of mass media campaign to reduce socioeconomic differences in women's awareness and behaviour concerning use of folic acid: cross sectional study. BMF 1999;319:291-2.

5 Cornel MC, Erickson JD. Comparison of national policies on periconceptional use of folic acid to prevent spina bifida and anencephaly (SBA). Teratology 1997;55:134-7. 